\title{
Query Evolution
}

\author{
W. Bruce Croft \\ Computer Science Department \\ University of Massachusetts Amherst \\ croft@cs.umass.edu
}

\begin{abstract}
Search engine queries have evolved over the past 30 years from complex Boolean formulations to short lists of "keywords." Despite the apparent simplicity of short queries, choosing the right keywords can be difficult, and understanding user intentions is a major challenge. Techniques such as query expansion and context-based profiles have been developed to address these problems, but with limited success. Rather than trying to infer user intentions from very short queries, another approach is to improve query processing and retrieval models for long queries. In particular, query transformation is a new approach to improving search that appears to have considerable potential. In this approach, queries are transformed into one or more new queries using probabilistic models for generation or search of query archives. I will describe various transformation models and the role of a retrieval model in using these transformations. Examples will be given from applications such as collaborative question answering and forum search.
\end{abstract}

